\title{
UN REFORMADOR DEL MUNDO: LA REPRESENTACIÓN PROPORCIONAL EN LA CORRESPONDENCIA DE JOHN STUART MILL (1859-1873)
}

\section{A Worldwide Reformer: Proportional Representation through John Stuart Mill's correspondence (1859-1873)}

\author{
SEBASTIÁN CORTESI' \\ Johns Hopkins University \\ Universidad de San Andrés \\ Universidad de Buenos Aires \\ sebastiangcortesi@gmail.com
}

Cómo citar/Citation

Cortesi, S. (2021).

Un reformador del mundo: la representación proporcional en la correspondencia de John Stuart Mill (1859-1873).

Revista de Estudios Políticos, 193, 317-337.

doi: https://doi.org/10.18042/cepc/rep.193.10

\section{Resumen}

La representación de las minorías ocupa un lugar central en la reflexión de John Stuart Mill acerca del gobierno representativo. Debido a ello, el lugar de Mill en la historia de los modernos regímenes electorales es objeto de polémica en la historiografía intelectual reciente. El presente artículo busca intervenir en dicho debate al argumentar que Mill ocupó un rol fundamental en el surgimiento de los sistemas electorales proporcionales. Además de proporcionar sólidos argumentos teóricos, Mill desplegó una verdadera campaña internacional a favor de la adopción de estos novedosos arreglos institucionales. Para demostrarlo, el artículo explora en profundidad su correspondencia personal, así como también otras fuentes primarias.

\footnotetext{
$1 \quad$ El autor agradece especialmente a Laura Cucchi sus valiosos comentarios a las versiones preliminares de este artículo.
} 


\section{Palabras clave}

John Stuart Mill; representación proporcional; sistemas electorales; conexiones transnacionales.

\section{Abstract}

Minority representation stands as a key topic in John Stuart Mill's reflections regarding representative government. As a consequence, his place in the history of modern electoral systems is a topic of debate in recent scholarship. This article argues that Mill played an instrumental role in the development of proportional electoral systems the world over. In addition to providing cogent arguments, he undertook an international campaign that advocated the adoption of these new institutional arrangements. His personal correspondence as well as other primary sources are explored in depth in order to probe so.

\section{Keywords}

John Stuart Mill; proportional representation; electoral systems; transnational connections. 


\section{SUMARIO}

I. DE LA «DEMOCRACIA PURA» A LA «DEMOCRACIA RACIONAL». EN BÚSQUEDA DEL SISTEMA ELECTORAL DEL FUTURO. II. UNA CAMPAÑA SIN FRONTERAS. III. LOS ECOS DE UNA PRÉDICA. BIBLIOGRAFía.

En su famoso ensayo sobre Tolstoi, Isaiah Berlin (2002: 35) sugiere que los grandes pensadores pueden ser calificados como zorros o erizos. Mientras que los zorros, amantes de lo particular, saltan de un tema a otro, los erizos tienen un principio o visión que organiza su obra. Como lo señaló Luis Blanc con motivo de su muerte, John Stuart Mill fue un personaje multifacético -filósofo, artista, economista y político-, para el cual la verdad científica fue un principio rector ${ }^{2}$. Mill, el «santo del racionalismo» según William Gladstone (Kinzer, 1981: 121), merece sin lugar a dudas un lugar junto a Platón, Hegel y Nietzsche en el panteón de los erizos ${ }^{3}$. Precisamente por ello su obra constituye terreno fértil para las indagaciones provenientes de la historia intelectual y de las ideas. Diversos estudios han explorado el modo en el cual Mill abordó problemáticas como la ampliación del sufragio y el colonialismo. Otros, además, han rastreado en dichos abordajes la influencia de autores como Tocqueville, Guizot y Carlyle (Pappe, 1964; Kinzer, 1981; Krouse, 1982; Bell, 2010; Miller, 2015; Paltieli, 2019).

Sin embargo, la contribución de Mill en el terreno de la política suele ser relegada como resultado del énfasis intelectual que atraviesa la historiografía reciente. La representación proporcional ilustra con claridad el argumento. La literatura ha aclarado las ideas de Mill acerca de la representación política y el lugar que esta ocupa en su reflexión madura sobre el gobierno representativo. En sus análisis, algunos autores sugieren que Mill debe ser considerado el padre intelectual de la representación proporcional mientras que otros, por el contrario, argumentan que sus ideas simplemente reflejan las inquietudes propias de los reformistas victorianos ante el advenimiento de la democracia (Kern, 1972; Hall, 1975; Pani, 2000:23; Conti, 2019). A pesar de divergir en su interpretación, ambos grupos coinciden en que sus investigaciones se focalizan únicamente en los libros, folletos y artículos periodísticos publicados por Mill.

2 The Standard, 22-07-1873

3 Las traducciones son propias a menos que se indique lo contrario. 
En línea con la primera posición, el presente artículo argumenta que Stuart Mill efectivamente desempeñó un rol central en el surgimiento de los sistemas electorales proporcionales. Además de aportar sólidos argumentos teóricos, Mill desplegó una campaña internacional para impulsar la adopción de estos novedosos arreglos electorales. Este trabajo indaga su correspondencia para demostrar que los vínculos establecidos con políticos, publicistas e intelectuales tanto británicos como foráneos contribuyeron a la formación de una red transnacional de actores dedicados a la difusión e implementación de nuevas fórmulas electorales (Rodogno et al., 2014; Haas, 1992). A su vez, la perspectiva adoptada permite apreciar el pragmatismo que caracterizó a Mill en su cruzada reformista, lo cual es soslayado por la historiografía intelectual al considerar que Mill depositó todas sus esperanzas en el sistema propuesto por el abogado y charity commissioner Thomas Hare (Urdánoz, 2008: 17; 2019).

El artículo contiene tres apartados. El primero describe brevemente el recorrido que condujo a Mill a concebir la representación de las minorías como la «hoja de ruta para la democracia del futuro» (Mikena y Lindley, 1972a: 765). El segundo reconstruye las acciones que Mill llevó a cabo para impulsar la adopción del nuevo principio de representación. Por último, el apartado final explora la recepción y el uso de su figura en algunos debates institucionales fuera de Gran Bretaña.

\section{DE LA «DEMOCRACIA PURA»A LA «DEMOCRACIA RACIONAL». EN BÚSQUEDA DEL SISTEMA ELECTORAL DEL FUTURO}

Desde un comienzo, las leyes electorales fueron para Mill un tema de primer orden. Con apenas diecisiete años, inició su vida pública definiéndose como un "partidario de una reforma radical» en la Cámara de los Comunes (Robson y Robson, 1986: 167). Para los radicales, tendencia en la cual su padre - James Mill-y Jeremy Bentham ocupaban un rol intelectual de relevancia, la ampliación de la franquicia era imperiosa ${ }^{4}$. La Constitución inglesa se hallaba desbalanceada en la medida que la mayoría de los comunes eran elegidos por unas pocas familias pertenecientes a la aristocracia (Robson y Stillinger, 1981: 78). Por lo tanto, ampliar el sufragio era la única forma de

4 Para los radicales utilitaristas la ampliación del sufragio debía ser complementada por la incorporación del voto secreto. Además, los mandatos parlamentarios debían ser anuales para asegurar una mayor coincidencia posible con el interés general (Duncan, 1969: 72). De lo contrario, la aristocracia venal continuaría imponiendo sus «intereses siniestros» por encima del interés general, lo cual suponía una amenaza para la libertad política. 
asegurar la identidad de intereses entre gobernantes y gobernados. Al igual que otros radicales, Mill estaba convencido de que la ampliación requerida iba más allá de lo que los Whigs estaban dispuestos a aceptar. Por ese motivo, en 1823 fundó la «radical y democrática» Westminster Review (Robson y Stillinger, 1981: 119). Editada por el propio Mill, la revista apuntaba a que los radicales ocuparan el lugar de los Whigs como partido impulsor de reformas. De ese modo, el ideal de progreso contaría con una debida expresión política en el Parlamento ${ }^{5}$ (Kinzer, 1981:110).

La apuesta fracasó. En vez de radicalizarse, los liberales colaboraron con los Tories y en 1832 la Great Reform Act amplió el electorado en un 45\% (Ertman, 2010: 1011) . Mill quedó decepcionado con buena parte de los radicales, a los cuales identificó como «metafísicos». Para estos, la ampliación de la franquicia era el resultado de premisas teóricas y no una solución al problema del buen gobierno (Hansson, 2013: 351). Para Mill, las clases trabajadoras eran tan egoístas como la aristocracia y, por lo tanto, su incorporación no pondría fin a la legislación de clase. Este dilema condujo al desarrollo de una teoría más exigente del gobierno representativo. Además de proteger el interés de los ciudadanos, este debía promover su desarrollo intelectual y moral (Krouse, 1982). A partir de 1830, Mill atravesó un breve período —influido por las lecturas de Saint Simon y Guizot - en el cual la dirección política de una élite instruida y desinteresada parece ser la clave de bóveda. Durante esos ańos, Mill participó activamente en la South Australian Association y su proyecto de crear una nueva colonia dirigida por legisladores ilustrados (Bell, 2010: 39)

Sin embargo, la experiencia al frente de la London and Westminster Review no fue en vano. En 1835 y 1840 respectivamente, Mill recibió y reseñó los dos volúmenes de «la primera investigación analítica acerca de las influencias de la democracia». La obra seminal de Tocqueville confirmó sus temores respecto de los dos grandes peligros de la democracia: la tiranía de la mayoría y la uniformidad de la opinión pública. Concomitantes al advenimiento de la sociedad democrática, estas amenazas son retratadas como fenómenos inexorables y de alcance global. Debido a ello, lo que Mill encuentra más valioso en la obra y asegura «dará un nuevo carácter a las especulaciones políticas de

5 Mill esperaba que algunos Whigs aceptaran el liderazgo radical y que los demás se afiliaran al bando Tory. De esta manera, los partidos finalmente pasarían a reflejar principios políticos claramente definidos e identificables.

6 De todos modos, la gran mayoría de la población adulta permaneció excluida del derecho al voto al no alcanzar el requisito de una renta anual de $£ 10$ - borough franchise-.

7 De hecho, el propio Mill consideró seriamente mudarse a Australia durante 1834. 
nuestro tiempo» es la idea de que las instituciones democráticas son capaces de corregir dichos defectos (Robson y Brady, 1977: 111). Parafraseando al propio Mill, las instituciones podrían suavizar los colores del cuadro (la democracia), pero no alterarlos (ibid.: 109). Por ejemplo, el sufragio indirecto matizaba la opinión y aseguraba la conformación de una Cámara de Senadores proba. Del mismo modo, las instancias representativas locales eran «laboratorios de cultura política» donde el pueblo podía participar y aprender a procurar el bien general (Cicalese, 2006: 51). En resumen, un buen diseño institucional permitía el desarrollo de una «democracia bien regulada»o "racional» en donde la igualdad política y el liderazgo de las minorías instruidas podían coexistir. De todos modos, no estaba claro cuál sería el sistema electoral capaz de lograr tan ambicioso objetivo.

En 1850, la ampliación del sufragio y la modificación de la legislación electoral ingresaron nuevamente en la agenda de los comunes. En un principio, Mill consideró que el voto plural —el otorgamiento de votos adicionales a los electores más capacitados - era una condición indispensable para cualquier ampliación de la franquicia ${ }^{8}$. Sin embargo, reconocía que la propuesta estaba en conflicto con el espíritu democrático (Mikena y Lindley, 1972a: 596). Esta intuición, compartida por varios reformistas victorianos, condujo a que se exploraran nuevas alternativas. Ante el imperativo de la inclusión, los políticos británicos evaluaron métodos capaces de garantizar la presencia de minorías en Westminster. Es decir, la representación de las minorías comenzó a ser concebida como un principio fundamental del gobierno representativo. La década de 1850 se debatió entre dos alternativas: el voto acumulativo y el voto limitado. El primero, propuesto por James G. Marshall (1853) y aplicado en las colonias de Malta (1849), Cabo Buena Esperanza (1850) y Bay Islands — actual Honduras(1856), contemplaba la posibilidad de que el elector otorgase todos sus votos a un único candidato. De esa manera, una minoría de electores tenía posibilidades de elegir a un representante en su distrito. En cambio, el voto limitado, defendido por Lord Russell, obligaba al elector a votar por menos candidatos que actas en juego para que la minoría obtuviera dicha diferencia.

Convencido de que una constitución justa requería que las minorías fueran representadas, Mill optó por la propuesta de Marshall en sus Thoughts

$8 \quad$ El esquema de voto plural propuesto por Mill (1859:20) otorgaba mayor peso a las preferencias de los electores más cultivados. Un trabajador ordinario dispondría de un solo voto, mientras que un trabajador calificado de dos. Luego, se le otorgarían tres o cuatro votos a los comerciantes, industriales y productores primarios. Finalmente, se contemplaba que los sectores más instruidos de la sociedad — graduados universitarios, empleados públicos y profesionales liberales- emitieran cinco o incluso seis sufragios. 
on Parliamentary Reform (1859). Esta resultaba atractivo debido a su simplicidad y su compatibilidad con el ideario liberal. Sin embargo, Mill no estaba del todo conforme. En una carta escrita en 1858 a Henry Chapman - colaborador de Mill en la London and Westminster Review y entonces miembro de la Cámara Alta del Parlamento de Victoria, Australia- Mill confesó lamentarse porque ninguno de los sistemas propugnados satisfacía el ideal de la democracia bien entendida, según el cual «cada porción del electorado debe poseer una influencia proporcional a su tamaño en la elección de representantes» (Mikena y Lindley, 1972a: 559).

\section{UNA CAMPAÑA SIN FRONTERAS}

Como bien ha señalado Gregory Conti (2019: 196), 1859 fue un año fundamental para John Stuart Mill. Además de su famoso ensayo On Liberty, en ese año hizo su aparición la primera edición del tratado de Thomas Hare sobre la elección de representantes ${ }^{9}$. En esa extensa e intrincada obra Mill finalmente encontró un sistema electoral capaz de «satisfacer los requisitos del principio (la representación de las minorías)» (Mikena y Lindley, 1972a: 606). Si bien su implementación era sencilla, el voto acumulativo y el voto limitado solamente permitían a la minoría acceder a una cuota fija y arbitraria de escaños. En cambio, la propuesta de Hare - un bill de 33 artículosprometía una asignación de escaños acorde al desempeño electoral mediante una sofisticada fórmula de cociente. En resumidas cuentas, la Cámara de los Comunes sería elegida en un único distrito nacional y cada elector emitiría un voto con sus candidatos en un orden descendente de preferencia ${ }^{10}$. Para resultar electos, los candidatos debían superar un umbral o cuota determinado por el cociente resultante de la división de los 658 escańos en juego por el total de electores inscriptos. Cuando un candidato superase la cuota, los votos sobrantes serían transferidos al candidato siguiente en el orden de preferencias de dichos votos sobrantes. De esta manera, Mill (1867: 9) estimó que unos tres mil votos bastarían para obtener un asiento en Westminster.

9 La obra $A$ Treatise on the Election of Representatives, Parliamentary and Municipal tuvo cuatro ediciones: 1859, 1861, 1865 y 1873 . Aunque lo cierto es que Hare presentó su propuesta de reforma electoral por primera vez en 1857 en un folleto titulado The Machinery of Representation.

10 En la propuesta inicial, el elector estaba habilitado para emitir tantas preferencias como escańos en juego. En otras palabras, el sistema de Hare contemplaba la posibilidad de papeletas con 658 candidatos. 
El tratado de Hare apareció en un momento oportuno. Mill compartió esta apreciación con el catedrático escocés y defensor del voto plural Alexander Bain. En Gran Bretaña, a diferencia de los Estados Unidos, «las mayorías numéricas no son aún la fuerza política predominante. La cuestión por decirse es cuánto poder habrá de serle otorgado» (Mikena y Lindley, 1972a: 606). Afortunadamente, buena parte del mundo - incluida Gran Bretaña - estaba a tiempo de adoptar instituciones capaces de prevenir este resultado. Mill no dudó en expresarle a Hare su entusiasmo: «Usted ha sido el primero en resolver con exactitud el problema de la representación popular y, al hacerlo, ha despejado la oscura nube de incertidumbre que pesaba sobre el futuro del gobierno representativo y por lo tanto de la civilización» (ibid.: 599).

A pesar de declararse como un "apóstol entusiasta» del cociente electoral, Mill desde un comienzo trazó objetivos realistas. El plan de Hare importaba una transformación radical de las instituciones electorales y por ello en una de sus primeras cartas Mill le advirtió que "una cosa tan completa no se obtendrá en un solo paso" (íd.). Si bien el voto limitado y el voto acumulativo eran apenas parches comparados con el cociente electoral, él habría de apoyarlos llegado el caso. En efecto, Mill confesó que no estaba dispuesto a «echar agua fría sobre aquello que reconoce un peligro y apunta en dirección al progreso" (Mikena y Lindley, 1972c: 1525). Adoptar una posición intransigente equivalía a no comprender la magnitud del desafío que los reformistas tenían por delante. «Nos encontramos en una carrera cuesta arriba, una carrera contra el tiempo», confesó a su amigo Henry Fawcett, «si la forma norteamericana de democracia nos alcanza primero, la mayoría no ejercerá una opresión menor a la de un único déspota» (Mikena y Lindley, 1972a: 672).

En 1860, Mill inició una activa campaña a favor de la reforma electoral. En primer lugar, buscó atraer el apoyo de figuras públicas y para ello envió copias del tratado de Hare a reformistas sociales como Edwin Chadwick, intelectuales de la talla de Herbert Spencer o James Lorimer, e influyentes políticos liberales como el futuro primer ministro William Gladstone (íd.). Además, procuró disponer de otras armas para dar la batalla en el terreno de la opinión. A pesar de su ingenio, Hare no dominaba «el arte de presentar sus descubrimientos con miras a causar un efecto en el público» (íd.). Por lo tanto, Mill encargó a Fawcett (1860) la confección de un folleto que fue publicado ese mismo año bajo el título Mr. Hare 's Reform Bill Simplified and Explained. Fue así cómo se inauguró todo un nuevo subgénero de la literatura política victoriana: los escritos dedicados a clarificar las ideas de Hare (Conti, 2019: 208). Por su parte, Mill también proporcionó una sólida defensa del sistema en su Considerations on representative government.

Para su agrado, la campańa adquirió estatus internacional prácticamente de inmediato. Una de las primeras noticias en este sentido provino de Adelaida 
—un municipio de la colonia australiana de Victoria-. Allí, en 1861, la prominente sufragista Catherine Helen Spence publicó varios artículos en el South Australian Gazette and Colonial Register reproduciendo los argumentos esgrimidos por Mill en una reseña de $1859^{11}$ (Hare, 1865: 323; Magarey, 2010). Dichos artículos fueron compilados y publicados nuevamente en un breve folleto polémico que fue remitido a Mill en 1862 por intermedio del político australiano Henry Parkes (Filipiuk y Laine, 1991: 137; Spence, 1861). Unos meses después, Mill expresaba a Thomas Hare la satisfacción que producía saber que la representación proporcional era materia de debate en Alemania y en Suiza, donde publicistas como Ernest Naville y Antoine Morin publicaban asiduamente sobre la materia (Mikena y Lindley, 1972a: 730, 779).

Del mismo modo, Mill felicitó a Luis Blanc, con quien mantenía una intensa correspondencia, por introducir en la opinión pública francesa el interrogante por las instituciones electorales adecuadas para una democracia libre (ibid.: 719). Además de Blanc, en Francia la representación proporcional contó con el apoyo de autoridades de la talla de Laboulaye, Eugène Véron y, en especial, Eugène Aubry-Vitet. De hecho, el artículo publicado por este último en la prestigiosa Revue des Deux Mondes fue considerado por Mill como una de las defensas más inteligentes y completas del sistema de Hare ${ }^{12}$. Obras como esta, argumentó, "propagarán el conocimiento y la apreciación (del sistema) a través de Francia y Europa de una manera muy eficaz» (Mikena y Lindley, 1972c: 1729). En efecto, esto fue precisamente lo que sucedió. En 1864, reformistas de toda Europa se reunieron en la ciudad de Ámsterdam con motivo del tercer congreso de la Asociación Internacional por el Progreso de las Ciencias Sociales (Carstairs, 2013; Müller, 2014). Luego de tres jornadas de deliberación, el comité apuntado para estudiar la «cuestión electoral» emitió un dictamen, redactado por el reformista belga M. Rolin-Jaequemyns, declarando al sistema electoral de Hare como el método más adecuado para asegurar «la libertad electoral y la sinceridad del voto» (Association Internationale pour le Progrès des Sciences Sociales, 1866: 213).

La representación de las minorías encontró defensores entusiastas incluso en los Estados Unidos, arquetipo de la democracia mayoritaria. Uno de ellos fue un arquitecto de Boston, Charles A. Cummings, quien en 1863 publicó una serie de artículos en el Christian Examiner (Mikena y Lindley, 1972a: 842). La proliferación de este tipo de notas periodísticas, que llegaban a Mill

11 El artículo titulado "Recent Writers on Reform» fue publicado en abril de 1859 en Fraser's Magazine.

12 El artículo de Eugène Aubry-Vitet fue publicado el 15 de mayo de 1870 bajo el tìtulo de «Le Suffrage Universel dans l'Avenir et le Droit de Représentation des Minorités». 
gracias al ministro unitario Daniel Conway, quien entonces se alojaba en casa de Elliot Cairnes, favoreció el desarrollo de conexiones trasatlánticas entre reformistas. Tanto Mill como Henry Fawcett, Walter Morrison, Richmond Droop e incluso el mismo Thomas Hare intercambiaron literatura e informes con sus pares americanos (Sterne, 1871: 10, 129; Dutcher, 1872: 86). Algunos de estos, como el abogado neoyorkino Dudley Field y el directivo de la Universidad de Harvard, William R. Ware, pautaron reuniones personales durante sus viajes a Europa (Mikena y Lindley, 1972a: 1342). Esperanzado por estos contactos, Mill comenzó a observar a los Estados Unidos bajo una luz diferente. Esta nueva perspectiva aparece con nitidez en una carta dirigida a Charles Cummings el 23 de febrero de 1863: «Sería digno de América inaugurar una mejora que es a la vez la aplicación más completa del principio democrático y, al mismo tiempo, su principal salvaguarda. Con el sistema de representación de todos en lugar de tan solo las mayorías [...] América ofrecería al mundo el primer ejemplo de verdadera igualdad democrática de la historia» (ibid:: 843).

Como sugiere la cita, Mill procuró que el nuevo principio de representación trascendiera la especulación teórica e ingresara en el terreno de las instituciones. Los esfuerzos en este sentido aparecen reiteradamente en su correspondencia y arrojan una imagen diferente a la del intelectual retraído en la torre de marfil. Una vez más, las colonias de Australia aparecieron como un espacio propicio para la innovación institucional. Como parte de un cambio de rumbo en la política del imperio, a partir de 1850 las colonias comenzaron a disponer de gobiernos responsables. Para Mill, esta era una oportunidad excepcional para llevar adelante experimentos que la metrópoli tenía vedados a causa de su rigidez y parroquialismo (Bell, 2010: 46). Además, las colonias australianas contaban con antecedentes en la materia. Rowland Hill —mejor conocido como el creador del sello postal — había ensayado un sistema similar al de Hare a nivel municipal durante su período al frente de la Comisión Colonizadora de South Australia (Mikena y Lindley, 1972a: 682). En mayo y agosto de 1861, dos proyectos de representación proporcional fueron presentados sin éxito ante el Parlamento de Victoria. Mill se mantuvo informado del trámite por medio de Henry Chapman, quien entonces integraba la Cámara Alta y además trabajaba como corresponsal del Times de Londres. En Victoria, los requisitos de propiedad establecidos por la Constitución de 1855 eran tan laxos que la Cámara Baja era elegida prácticamente por sufragio universal (ibid.: 764). Por tanto, la colonia reunía las condiciones necesarias para convertirse en un caso modelo:

El curso de la política bajo su actual constitución es precisamente aquel de la falsamente llamada democracia en la cual los trabajadores son los únicos 
realmente representados. Las viejas naciones se encontrarán con el tiempo en la misma situación, y la única forma de evitarlo es luchar con coraje, como ustedes lo hacen [...] luchar siempre por una justa representación de las minorías. Yo veo en ella la hoja de ruta para la democracia del futuro. Si no es adoptada, nada evitará que la sociedad sea conducida a un nivel mental de estrecha estupidez y a la anarquía (ibid.: 765$)^{13}$.

Cuando en 1863 un nuevo proyecto buscó introducir el voto acumulativo en Victoria, los parlamentarios resaltaron que el autor del mismo había sido contactado y felicitado por el mismísimo John Stuart Mill (Hare, 1865: 327). Al igual que con Chapman, Mill mantuvo contacto con otro compatriota emigrado a Australia y convertido en legislador. George Holden, miembro de la Cámara Alta del Parlamento de South New Wales, fue uno de los principales impulsores del sistema diseñado por Hare e, incluso, citó extensamente pasajes de las Considerations on Representative Government durante su exposición en el recinto (Hare, 1865: 311). Desafortunadamente, el final del ministerio de William Forster alteró el panorama político y como consecuencia el proyecto jamás fue tratado por la Cámara revisora. Unos meses después, Mill fue sorprendido por una carta de Robert Crichton Wyllie - ministro de Relaciones Exteriores del Reino de Hawai-, quien le informaba acerca de sus planes para adoptar la representación proporcional (Mikena y Lindley, 1972a: 834). A pesar de que ninguno de estos proyectos prosperó, los debates coloniales arrojaban para Mill un saldo positivo. A diferencia de Westminster, donde la cuestión no había logrado siquiera ingresar al recinto, en las colonias había "menos prejuicio contra las novedades" y ello permitía que el asunto fuese debidamente examinado. En las colonias, «los conservadores apoyan el plan como un modo de limitar el poder absoluto de las mayorías numéricas, y los demócratas porque es un corolario directo y obvio del principio democrático" (ibid.: 940).

Mill no se dio por vencido; por el contrario, las elecciones de 1865 le dieron la oportunidad de abogar por la representación de las minorías como miembro de la Cámara de los Comunes en un contexto en el cual la reforma electoral ocupaba el centro de la escena. Sin embargo, él no aprovechó esta oportunidad hasta mayo de 1867. Durante buena parte de su mandato, Mill se vio forzado a adoptar una postura moderada como consecuencia de su apoyo a William Gladstone. La muerte de Palmerston había permitido su llegada al ministerio y Mill consideró que desde esa posición conduciría al partido liberal por un rumbo verdaderamente reformista (Kinzer, 1981: 114).

13 El resaltado es propio. 
En consecuencia, Mill dedicó varias de sus intervenciones a respaldar un proyecto de reforma que en privado describió como «una especie de improvisación que para personas de mi opinión es difícil de defender» (Mikena y Lindley, 1972b: 1169). La esperanza depositada en Gladstone incluso lo condujo a rechazar la propuesta de Earl Grey de incluir el voto acumulativo en el proyecto oficial ${ }^{14}$.

La llegada del Gobierno conservador de Derby marcó un punto de inflexión. A partir de entonces, Mill defendió públicamente la representación proporcional a pesar de que «todos excepto nosotros (Mill y Hare) y los radicales extremos se le oponen" (ibid.: 1244). En su famoso discurso del 29 de mayo 1867, Mill (1867: 16) argumentó ante los Comunes que él no se encontraba solo en su prédica; por el contrario, el sistema diseñado por Hare contaba entre sus defensores a «los principales escritores y figuras públicas de Alemania, Francia, Suiza, Italia, nuestras colonias australianas y los Estados Unidos». Si bien la propuesta no prosperó, Mill encargó la publicación de su discurso, al cual anexó publicaciones, artículos y debates legislativos de cada uno de esos países.

Entre estos casos, Suiza destacó por ofrecer un caso práctico. Allí había un intelectual y publicista que compartía su visión sobre «una de las cuestiones más serias de la ciencia política». El catedrático Ernest Naville —apodado el John Stuart Mill continental (Maglie, 2014: 55)— contribuyó de forma decisiva al movimiento reformista tanto en su país como en otras latitudes al fundar en 1865 la Association Réformiste de Ginebra. Esta organización se dedicó a impulsar proyectos de reforma y defender la representación proporcional mediante su periódico semanal La Réformiste. Cantón tras cantón, estas asociaciones se reprodujeron y, en 1876, se fusionaron en una única organización nacional. Con la ayuda de Edmond Beales, presidente de la Reform League, Mill y Hare dotaron a Gran Bretańa de su propia asociación reformista: la Representative Reform Association. La misma fue el resultado de cuatro reuniones que tuvieron lugar en la sede de la Reform League entre el 28 de febrero y el 21 de marzo de 1868 (Parsons, 2009: 111). El listado de invitados refleja la ambición global que caracterizó los esfuerzos de Mill. Además de parlamentarios afines como Henry Fawcett y Thomas Hughes, la asociación fue integrada prácticamente por todos los reformistas foráneos con los que había intercambiado correspondencia: G. K. Holden (South New Wales), C.H. Spence (South Australia), Dudley Field (Estados Unidos), Ernest Naville (Suiza), Rolin-Jaequemyns (Bélgica), entre otros.

14 Mill rehusó a ser quien presentase la enmienda. Sin embargo, esta habría de contar con su voto en el hipotético caso de que otro parlamentario la presentase. 
Entre sus objetivos, la Representative Reform Association se propuso examinar, comparar y discutir sistemas electorales, presentarlos ante la opinión pública y, por último, funcionar como «centro de comunicación con la Personal Representation Society de Nueva York, la Association Réformiste de Ginebra y las demás sociedades y personas con propósitos similares» (Representative Reform Association, 1868: 1).

La prédica de Mill no resultó en vano. Si bien los Comunes desestimaron el cociente de Hare, lo cierto es que la Second Reform Act (1867) contempló la representación de las minorías en los grandes distritos urbanos de Inglaterra mediante el voto limitado. A decir verdad, la medida en cuestión era extremadamente moderada, dado que estos catorce distritos representaban apenas 40 de los 658 escaños de Westminster. Además, como bien señaló uno de los traductores de Mill, el profesor de Derecho Constitucional colombiano Florentino Gonzáles (1871:184), estos distritos pertenecían por completo al liberalismo. En otras palabras, el voto limitado otorgó a los conservadores escaños que no habrían podido obtener de otro modo. Aún así, Mill celebró la reforma porque vio en ella una concesión al principio y un valioso expediente práctico. Así se lo transmitió a su colega reformista y defensor del voto acumulativo, James G. Marshall: «Por pequeña que sea, la base que el principio ha obtenido en nuestra legislación gracias a la nueva Ley de Reforma asegura que la cuestión seguirá siendo discutida una y otra vez con objetivos prácticos» (Mikena y Lindley, 1972b:1322).

\section{LOS ECOS DE UNA PRÉDICA}

A diferencia de su faz intelectual, la acción política de John Stuart Mill se encuentra atravesada por apuestas fallidas y proyectos truncados. De hecho, él mismo era consciente de este contraste. En una carta dirigida a Thomas Hare, Mill buscaba consuelo en el hecho de que «aunque los políticos se alejan gustosos del asunto, las ideas correctas, ahora que son promulgadas, están rápidamente abriéndose paso entre los pensadores, los futuros maestros de todas partes del mundo" (Mikena y Lindley, 1972a: 779). Como buen erizo, Mill estaba convencido de la pertinencia de su principio y, a pesar de los fracasos parlamentarios, no perdió la esperanza. «Comienzo a pensar», confesó a Hare en 1866, "que tú o yo viviremos para ver el plan en funcionamiento en Inglaterra o en todo caso en América» (Mikena y Lindley, 1972b: 1132).

Aunque no lo vio en vida, Mill estaba en lo correcto al vincular la representación proporcional con el futuro de la democracia. Comenzando por Bélgica en 1899, prácticamente toda Europa Occidental acompañó la ampliación del sufragio con la adopción de sistemas electorales proporcionales durante 
las dos primeras décadas del siglo xx (Negretto y Visconti, 2018). De hecho, los estudios revelan que los sistemas electorales creados o reformados durante la primera mitad del siglo pertenecen a la familia proporcional (Calvo, 2009). Del mismo modo, un gran número de naciones de Europa Oriental se incorporaron a la familia luego de 1989 (Williams, 2003). En resumen, es seguro afirmar que la amplia mayoría de las democracias contemporáneas utilizan algún tipo de procedimiento proporcional para asignar actas legislativas.

Este artículo argumenta que Stuart Mill contribuyó significativamente al surgimiento y expansión de estos sistemas. Por un lado, proporcionó sólidos argumentos teóricos en favor de la reforma en sus obras, así como también en sus discursos y otras intervenciones públicas. Además, incentivó y colaboró en diversos intentos de reforma en Gran Bretaña y en el extranjero, sirviéndose para ello de sus contactos internacionales. Pero, sobre todo, Mill merece un lugar en el panteón de los padres del movimiento internacional proporcionalista porque así fue reconocido por sus contemporáneos, incluso aquellos con los cuales jamás intercambió correspondencia. A modo de cierre, esta sección ofrece algunos ejemplos ilustrativos de la recepción y el uso de la figura de John Stuart Mill en diferentes movimientos reformistas.

Como hemos visto, Mill celebró el progreso de la representación de las minorías en los Estados Unidos. De aquel lado del Atlántico, las propuestas de reforma comenzaron a multiplicarse tanto a nivel federal como estadual durante el periodo inmediatamente posterior a la guerra civil. Una de las principales iniciativas fue encabezada por el senador por Pensilvania, Charles R. Buckalew, quien en 1867 propuso abandonar el sistema de circunscripciones uninominales para la Cámara de Representantes y, en su lugar, adoptar el voto acumulativo. Buckalew obtuvo un dictamen de comisión favorable y el 11 de julio se dirigió al Senado. En su discurso, el cual fue publicado y llegó a manos de Mill por medio de James G. Marshall, el senador citó extensivamente a la «máxima autoridad en la materia» para demostrar que el proyecto era conveniente, practicable y útil (Mikena y Lindley, 1972b: 1244; Buckalew, 1872: 27). Aunque su propuesta no prosperó, Buckalew logró convencer de los beneficios del voto acumulativo al prominente republicano y editor del Chicago Tribune Joseph Medill (ibid:: 166). Este entusiasta defensor de la representación de las minorías fue electo convencional constituyente del estado de Illinois en noviembre de 1869 y designado presidente del Comité de Reformas Electorales y Representativas. Al igual que Buckalew, Medill recurrió a la autoridad de los argumentos expuestos en la Cámara de los Comunes en 1867 por «uno de los más grandes exponentes de la ciencia social y política» (Illinois Constitutional Convention, 1870: 1727).

Sin embargo, en esta ocasión la iniciativa recibió el respaldo de demócratas y republicanos. Una vez culminadas las deliberaciones, el diario de 
Medill y la recientemente formada Chicago Minority Representation Society desplegaron una campaña que contribuyó a que la reforma finalmente se impusiera en las urnas el 2 de julio de $1870^{15}$. Illinois se convirtió así en el primer estado en elegir la totalidad de sus representantes mediante un régimen electoral con representación de minorías. De hecho, en su Historia Constitucional de Illinois el convencional Elliot Anthony (1891: 121) señala que «la representación de las minorías era, al momento de su introducción, una novedad en la ciencia política, y considerada como experimental». Si bien Mill no se refirió al asunto, su correspondencia sugiere que estuvo al tanto de los acontecimientos de Illinois gracias a un informe - La question électorale en Europe et en Amérique: Rapport présenté à l'Association Réformiste de Genèveenviado por su propio autor, el ginebrino Ernest Naville (Filipiuk y Laine, 1991:189).

A su vez, la figura de Mill fue utilizada en muchos debates institucionales de los cuales no hay evidencia de que haya estado al tanto. El caso de las repúblicas hispanoamericanas ilustra bien el argumento. Por ejemplo, los argumentos presentados en los capítulos siete y ocho de las Consideraciones sobre el gobierno representativo fueron debatidos en la Cámara de Diputados del imperio brasilero en el mismo año de su publicación. De hecho, uno de los miembros más conspicuos del cuerpo, José de Alencar, se definía como «un soldado de Stuart Mill» (Rizzo, 2012: 39). Del mismo modo, en Chile la obra de Mill era leída por el club de la reforma de la capital ${ }^{16}$. En 1869, este club electoral incluyó el voto acumulativo en su programa político, organizó conferencias dedicadas a la cuestión e incluso lo adoptó como mecanismo para la designación de su propio personal directivo ${ }^{17}$.

En una carta dirigida a Luis Blanc, Mill aseguró que «las buenas ideas, una vez plantadas, echan raíces en la tierra». Lamentablemente, Mill no vivió para ver florecer la representación proporcional. En junio de 1873 su médico le informó de que su condición era terminal. Llamativamente, el diario porteńo editado en inglés coincidió con el tono de la reflexión del propio Mill al aseverar que «las semillas que ha ayudado a plantar están comenzando a brotar por doquier ${ }^{18}$. En efecto, en esos meses la convención constituyente de Buenos Aires se encontraba debatiendo el futuro del régimen representativo

15 Los convencionales constituyentes sometieron la reforma electoral a un voto separado del resto del texto constitucional. Como bien ha seńalado Frank Cicero Jr. (2018:175), este era un procedimiento habitual cuando se trataba de asuntos polémicos y que por ende podían poner en peligro la aprobación de la nueva constitución estadual.

16 Mercurio de Valparaíso, 3-9-1869

17 Mercurio de Valparaiso, 7-9-1883

18 The Standard, 15-6-1873 
de la provincia. Una vez más, «Stuard Mill [sic], que es uno de los partidarios de este bello sistema de la representación proporcional» se hizo presente en el recinto (Varela, 1877: 213). Como bien ha señalado Leonardo Hirsch (2018), el constituyente bonaerense —influido por las obras de Hare y Mill- fue el primero del mundo en consagrar la proporcionalidad como principio de representación; es decir, como "regla en todas las elecciones populares» ${ }^{19}$.

\section{Bibliografía}

Anthony, E. (1891). The Constitutional History of Illinois. Chicago: Chicago Legal News Print. Association Internationale pour le Progrès des Sciences Sociales (1866). Annales de L'Association Internationale pour le Progrès des Sciences Sociales. Quatrième Session. Paris: Guillamauin and Co.

Bell, D. (2010). John Stuart Mill on Colonies. Political Theory, 38 (1), 34-64. Disponible en: https://doi.org/10.1177/0090591709348186.

Berlin, I. (2002). El erizo y la zorra. Tolstoi y su visión de la historia. Barcelona: Península.

Buckalew, C. R. (1872). Proportional Representation: Or The Representation of Successive Majorities in Federal, State, Municipal, Corporate and Primary Elections. Philadelphia: J. Campbell and Son.

Calvo, E. (2009). The competitive road to proportional representation. World Politics, 61, 254. Disponible en: https://doi.org/10.1017/S0043887109000100.

Carstairs, A. M. (2013). A short history of electoral systems in Western Europe (vol. 22). New York: Routledge.

Cicalese, M. L. (2006). Centralisation y Liberty: reflexiones de John Stuart Mill. Anuario de Filosofia del Derecho, 23, 37-60.

Cicero Jr, F. (2018). Creating the Land of Lincoln: The History and Constitutions of Illinois, 17781870. Illinois: University of Illinois Press. Disponible en: https://doi.org/10.5406/j. ctt21h4xzh.

Conti, G. (2019). Parliament the mirror of the nation: Representation, deliberation, and democracy in Victorian Britain. Cambridge: Cambridge University Press. Disponible en: https://doi.org/10.1017/9781108582469.

Duncan, G. (1969). John Stuart Mill and Democracy. Politics, 4 (1), 67-83. Disponible en: https://doi.org/10.1080/00323266908401157.

Dutcher, S. (1872). Minority Or Proportional Representation: Its Nature, Aims, History, Processes, and Practical Operation. New York: United States Publishing Company.

Ertman, T. (2010). The Great Reform Act of 1832 and British Democratization. Comparative Political Studies, 43 (8-9), 1000-1022. Disponible en: https://doi.org/10.1177/001 0414010370434.

Fawcett, H. (1860). Mr. Harés Reform Bill Simplified and Explained. London: J. Ridgway.

19 Constitución de la Provincia de Buenos Aires (1873). Edición Oficial. Buenos Aires: Imprenta Americana. 
Filipiuk, M. y Laine M. (eds.) (1991). The Collected Works of John Stuart Mill, Volume XXXII. Additional Letters of John Stuart Mill. Toronto: University of Toronto Press.

González, F. (1871). Lecciones de Derecho Constitucional. Segunda versión corregida y aumentada. Paris: Rosa y Bouret.

Haas, P. M. (1992). Introduction: epistemic communities and international policy coordination. International Organization, 1-35. Disponible en: https://doi.org/10.1017/ S0020818300001442.

Hall, D. D. (1975). The Victorian Connection. American Quarterly, 27 (5), 561-574. Disponible en: https://doi.org/10.2307/2712441.

Hansson, S. O. (2013). John Stuart Mill's political self-identifications. Journal of Political Ideologies, 18 (3), 348-357. Disponible en: https://doi.org/10.1080/13569317.2013.831593.

Hare, T. (1857). The machinery of representation. London: W. Maxwell.

- (1865). A treatise on the election of representatives, parliamentary and municipal. London: Longman; Green; Longman and Roberts.

Hirsch, L. D. (2018). La representación proporcional contra los comités. Un análisis sobre las fuentes político-intelectuales que inspiraron el debate en torno al régimen electoral de la provincia de Buenos Aires (1870-1876). Boletín del Posgrado en Historia de la Universidad Torcuato Di Tella.

Illinois Constitutional Convention (1870). Debates and Proceedings of the Constitutional Convention of The State of Illinois, Convened at the city of Springfield, Tuesday, December 13, 1869. Volume II. Springfield: E. L. Merritt and Brother.

Kern, P. B. (1972). Universal Suffrage without Democracy: Thomas Hare and John Stuart Mill. The Review of Politics, 34 (3), 306-322. Disponible en: https://doi.org/10.1017/ S0034670500026097.

Kinzer, B. L. (1981). JS Mill and the Problem of Party. The Journal of British Studies, 21 (1), 106-122. Disponible en: https://doi.org/10.1086/385785.

Krouse, R. W. (1982). Two concepts of democratic representation: James and John Stuart Mill. The Journal of Politics, 44 (2), 509-537. Disponible en: https://doi.org/10.2307/2130598.

Magarey, S. (2010). Unbridling the Tongues of Women: A Biography of Catherine Helen Spence. Adelaida: University of Adelaide Press. Disponible en: https://doi.org/10.1017/ UPO9780980672305.

Maglie, D. (2014). Le origini del movimento proporzionalista in Italia e in Europa con particolare riguardo alle associazioni sorte in Svizzera e Belgio [tesis doctoral]. Universidad Roma Tre.

Mikena, E. F. y Lindley, D. (eds.) (1972a). The Collected Works of John Stuart Mill, Volume XV. The Later Letters of John Stuart Mill 1849-1873. Part II. Toronto: University of Toronto Press.

- (1972b). The Collected Works of John Stuart Mill, Volume XVI. The Later Letters of John Stuart Mill 1849-1873. Part III. Toronto: University of Toronto Press

- (1972c). The Collected Works of John Stuart Mill, Volume XVII. The Later Letters of John Stuart Mill 1849-1873. Part IV. Toronto: University of Toronto Press.

Mill, J. S. (1859). Thoughts on parliamentary reform. London: John W. Parker and Son.

- (1865). Considerations on representative government. London: Longman; Green; Longman, Roberts and Green. 
(1867). Personal Representation. Speech of John Stuart Mill delivered in the House of Commons, May 29th, 1867. With an appendix containing notices of reports, discussions and publications on the system in France, Geneva, Germany, Belgium, Denmark, Sweden, The Australian Colonies and The United States. London: Henderson; Rait and Fenton.

Miller, D. E. (2015). The Place of Plural Voting in Mill's Conception of Representative Government. The Review of Politics, 399-423. Disponible en: https://doi.org/10.1017/ S0034670515000340.

Müller, C. (2014). The Politics of Expertise: The Association Internationale pour le Progrès des Sciences Sociales. Democratic Peace Movements and International Law Networks in Europe 1858-75. En D. Rodogno, B. Struck y J. Vogel (eds.). Shaping the Transnational Sphere: Experts, Networks, and Issues (c. 1850-1930). New York: Berghahn Books.

Negretto, G. L. y Visconti, G. (2018). Electoral Reform Under Limited Party Competition: The Adoption of Proportional Representation in Latin America. Latin American Politics and Society, 60 (1), 27-51. Disponible en: https://doi.org/10.1017/lap.2017.5.

Paltieli, G. (2019). Mill's closet: JS Mill on solitude and the imperfect democracy. History of European Ideas, 45 (1), 47-63. Disponible en: https://doi.org/10.1080/01916599.2018 .1527558 .

Pani, E. (2000). En busca de la «democracia real». Prismas-Revista de Historia Intelectual, 4 (1), 21-46.

Pappe, H. O. (1964). Mill and Tocqueville. Journal of the History of Ideas, 25 (2), 217-234. Disponible en: https://doi.org/10.2307/2708013.

Parsons, F. D. (2009). Thomas Hare and political representation in Victorian Britain. Basingstoke: Palgrave Macmillan. Disponible en: https://doi.org/10.1057/9780230244665.

Representative Reform Association (1868). Representative Reform Report of the committee appointed by the conference of members of The Reform League, and others, on Mr. Hare's Scheme of Representation. London: Trubner and Co.

Rizzo, R. (2012). Sobre rochedos movediços: deliberação e hierarquia no pensamento político de José de Alencar. São Paulo: Hucitec.

Robson, A. P. y Robson, J. M. (eds.) (1986). The Collected Works of John Stuart Mill, Volume XXII. Newspaper Writings December 1822-July 1831. Part I. Toronto: University of Toronto Press.

Robson, J. M. y Brady, A. (eds.) (1977). The Collected Works of John Stuart Mill, Volume XVIII. Essays on Politics and Society. Part I. Toronto: University of Toronto Press.

Robson, J. M. y Stillinger, J. (eds.) (1981). The Collected Works of John Stuart Mill, Volume I. Autobiography and Literary Essays. Toronto: University of Toronto Press.

Rodogno, D., Struck, B. y Vogel, J. (eds.). (2014). Shaping the Transnational Sphere: Experts, Networks and Issues from the 1840s to the 1930s. New York: Berghahn Books.

Spence, C. H. (1861). A Plea for Pure Democracy: Mr. Hare's Reform Bill Applied to South Australia. Melbourne: W. C. Rigby and George Robertson.

Sterne, S. (1871). On Representative Government and Personal Representation: Based in Part Upon Thomas Hare's Treatise, Entitled «The Election of Representatives, Parliamentary and Municipal». Philadelphia: JB Lippincott and Company.

Urdánoz, J. (2008). ¿Defendió Stuart Mill la representación proporcional? Notas contra un paradigma. Revista de Estudios Políticos, 139, 13-44. 
- (2019). John Stuart Mill and proportional representation. A misunderstanding. Political Science, 71 (2), 158-171. Disponible en: https://doi.org/10.1080/00323187.2019.170 1949.

Varela, L. V. (1877). Debates de la Convención Constituyente de Buenos Aires 1870-1873. Tomo II. Buenos Aires: La Tribuna.

Williams, K. (2003). Proportional representation in post communist Eastern Europe: the first decade. Representation, 40 (1), 44-54. Disponible en: https://doi.org/10.1080/00344 890308523245 . 